\title{
La Comunicacion en Galicia 2000-2003: Realidades y Sueños
}

\author{
Dr. Xosé López \\ Profesor Titular de Periodismo del Departamento de Ciencias de la Comunicación. \\ Facultad de Ciencias de la Información de la Universidad de Santiago de Compostela
}

\section{RESUMEN}

Galicia ha entrado en el siglo XXI con un sistema de medios de comunicación que se caracteriza por el predominio de la empresa de propiedad familiar, por una cierta debilidad empresarial (incremento de la deuda y dependencia de las subvenciones), por el liderazgo en el mercado local y regional frente a los medios de Madrid, por una estrategia de atención a los contenidos de proximidad y por la escasa presencia de la lengua propia en los contenidos de la mayoría de los productos de las empresas privadas. Es un modelo paradigmático de medios locales que a lo largo de la historia ha conseguido sentar las bases para articular una fuerte implantación en los ámbitos de proximidad y una aceptable sintonía con los usuarios en un mercado presidido por una tendencia a la estabilidad (los índices de difusión y audiencia se han estabilizado desde 1995). El último tercio del siglo $X X$ ha sido de grandes cambios en la mayoría de las empresas, que han aprovechado el año 2000, 2001 y 2002 para renovar varios proyectos periodísticos y para proseguir con la política de diversificación y de creación de grupos de comunicación capaces de ganar en tamaño y en estabilidad en el escenario de la Sociedad de la Información. Los datos apuntan que en el año 2003 la industria cultural y del conocimiento facturará alrededor de los cuatrocientos mil millones de pesetas, de los que una tercera parte corresponde a la actividad económica de la prensa escrita, del sector audiovisual y del negocio editorial. La facturación de la empresa periodística gallega superó los treinta mil millones de pesetas.

\section{ABSTRACT}

Galicia has entered XXI century with a media system characterised by the predominance of familiar companies, a certain managerial debility (increasing of debt and dependence on subsidies), the leadership of regional and local media over Madrid's ones, the attention to proximity contents as a strategy of communication and the scarce use of Galician language in most of the products of private companies.

It is a paradigmatic model of local media that achieved a great implantation in the proximity area and an acceptable relation with its audience in a market with tendency to stability (data of diffusion and audience are regular since 1995).

In the last decades of XXI century there were many changes in most of the companies. During the years 2000, 2001 and 2002, these companies reorganised several journalism projects and carried on a model of diversification and creation of communication groups, in order to acquire stability and to increase their size in the Information Society.

Facts point out that on 2003 culture industry will turn over around four hundred thousand million of pesetas, from which a third part corresponds to the activity of the press, the audio-visual sector and the publishing area. The turnover of the Galician press business surpassed thirty thousand millions of pesetas.

Palabras claves: Comunicación local/Estructura de la información/Periodismo.

Key words: Communication in Galicia 2000-2003/Realities and dreams. 


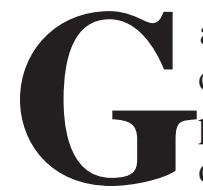
alicia ha entrado en el siglo XXI con un sistema de medios de comunicación que se caracteriza por el predominio de la empresa de propiedad familiar, por una cierta debilidad empresarial (incremento de la deuda y dependencia de las subvenciones), por el liderazgo en el mercado local y regional frente a los medios de Madrid, por una estrategia de atención a los contenidos de proximidad y por la escasa presencia de la lengua propia en los contenidos de la mayoría de los productos de las empresas privadas. Es un modelo paradigmático de medios locales que a lo largo de la historia ha conseguido sentar las bases para articular una fuerte implantación en los ámbitos de proximidad y una aceptable sintonía con los usuarios en un mercado presidido por una tendencia a la estabilidad (los índices de difusión y audiencia se han estabilizado desde 1995). El último tercio del siglo XX ha sido de grandes cambios en la mayoría de las empresas, que han aprovechado el año 2000, 2001 y 2002 para renovar varios proyectos periodísticos y para proseguir con la política de diversificación y de creación de grupos de comunicación capaces de ganar en tamaño y en estabilidad en el escenario de la Sociedad de la Información. Los datos apuntan que en el año 2003 la industria cultural y del conocimiento facturará alrededor de los cuatrocientos mil millones de pesetas, de los que una tercera parte corresponde a la actividad económica de la prensa escrita, del sector audiovisual y del negocio editorial. La facturación de la empresa periodística gallega superó los treinta mil millones de pesetas.

El espacio de comunicación de Galicia atraviesa momentos de gran vitalidad como lo prueba el incremento de cabeceras de diarios, la creación de nuevas emisoras de radio y televisión local y la aparición de medios en línea. Los medios gallegos aumentan su presencia en un territorio donde los ciudadanos eligen lo propio frente a lo foráneo en los medios impresos, mientras hacen lo contrario en los medios audiovisuales y las otras industrias culturales. Los canales de televisión española y la emisión en cadena de las radios estatales mantienen la hegemonía en un momento en que las televisiones y las radios locales ganan audiencia, y Televisión de Galicia avanza con la pretensión de invertir una tendencia consolidada durante los años de televisión única. Lo gallego despierta interés en Galicia, que tiene menos de tres millones de habitantes, aunque en los medios de comunicación lo hace casi siempre de la mano de la lengua castellana.

\section{GALICIA}

\section{CIFRAS OFICIALES DE POBLACIÓN}

\begin{tabular}{|lcccc|}
\hline & Población a 01/01/00 & Población a 01/01/01 Variación absoluta & Variación relativa \\
A Coruña & 1.108 .419 & 1.108 .002 & -417 & $-0,04$ \\
Lugo & 365.619 & 364.125 & -1.494 & $-0,41$ \\
Ourense & 345.241 & 344.623 & -618 & $-0,18$ \\
Pontevedra & 912.621 & 916.176 & 3.555 & 0,39 \\
Galicia & 2.731 .900 & 2.732 .926 & 1.026 & 0,04 \\
\hline
\end{tabular}

Fuente: Instituto Galego de Estatística 
El dinamismo del año 2000 estuvo marcado por la alargada sombra del aniversario de los doscientos años de la publicación de El Catón Compostelano, el primer diario de Galicia, ahora recuperado en edición facsimilar por el Consello da Cultura Galega y para el patrimonio público. La única edición completa que se conoce estaba en manos privadas y ha sido adquirida por la Consellería de Cultura, Comunicación Social y Turismo para la hemeroteca de la Cidade da Cultura, el ambicioso proyecto del Gobierno gallego que albergará la gran biblioteca de Galicia, el museo de Galicia y un gran centro de nuevas tecnologías y audiovisual del que en febrero del 2001 se colocó la primera piedra.

Mientras los investigadores analizaban el contexto en el que apareció El Catón Compostelano y las características de este soporte impreso, los diarios de Galicia aprovechaban las celebraciones para poner en marcha nuevos proyectos dirigidos a la consolidación de los grupos editores y a la diversificación de productos. Las iniciativas llegaron de la mano de nuevos directores que asumieron la responsabilidad de conducir por el camino del éxito a veteranos productos en el mercado periodístico.

La lista de relevos la inició Diario de Pontevedra, donde el cambio de propiedad puso fin a la única cooperativa de producción que existía en nuestro ámbito, sustituida por una sociedad limitada, y colocó al frente del diario al profesor universitario y periodista Antón Galocha. Era el mes de marzo de 2000. En el mes de junio, La Voz de Galicia, una de las cabeceras centenarias y la de mayor difusión, cambia de director. Bieito Rubido, director adjunto, sustituye a Ernesto Sánchez Pombo, que pasa a director de proyectos editoriales.

La empresa Rías Baixas Comunicación, editora de Atlántico Diario, nombró en noviembre del año 2000 a Fernando Ojea como director y decidió que el director saliente, Rodrigo Varela, pasase a ser asesor editorial del Grupo La Región. El Progreso, diario de Lugo, relevó a Fernando Salgado en la dirección el 20 de diciembre del 2000. El puesto lo ocupó Luis Rodríguez García, más conocido por "Lois Caeiro".

Los otros tres periodistas que estrenaron dirección de diarios en fechas recientes han sido Germán Castro, director de Diario de Ferrol desde su nacimiento (en junio de 1999); Francisco Orsini, director de La Opinión de A Coruña desde el primer número, en octubre del 2000; y Antón Xil, director de Diario de Arousa desde febrero del 2001, fecha de su aparición en el mercado. En total siete cambios -cuatro relevos y tres nombramientos para nuevas cabeceras- en la dirección informativa de los doce diarios de información general que se editan en Galicia.

La cabecera número trece de esta relación que completa el panorama de diarios, Deporte Campeón, es un deportivo editado en A Coruña, que ha visto desaparecer a su competidor local, Meta Sport (cerrado en el verano de 1999), y que ha estrenado director en agosto del 2000, Juan Carlos Boga, el ex redactor jefe 
que recogió el relevo del anterior responsable periodístico del primer diario deportivo de Galicia, el coruñés César Casal.

La incorporación de caras nuevas al frente de los equipos directivos de las redacciones de los periódicos gallegos finalizó, en la etapa analizada, el 1 de abril de 2001 con el nuevo organigrama de redacción de La Voz de Galicia. En esta ocasión el diario coruñés promocionó a sus periodistas con el nombramiento de Xosé Luis Vilela como director adjunto; Alfredo Vara, como subdirector de fin de semana; César Casal, como subdirector de información local; Luis Ventoso, como subdirector de apertura; y Carlos Agulló, como subdirector de cierre. A la jefatura de área de Galicia se promovió a Lois Blanco, a la de actualidad a Javier Armesto, a la de sociedad a Jorge Casanova y a la de deportes a Fernando Hidalgo. A la lista de redactores jefes se incorporaron José Varela y Lalo Fernández.

Los once nombramientos se produjeron poco después de que Santiago Rey Fernández-Latorre apartase a sus hijos de la gestión del Grupo Voz y de que se anunciase la aparición de un nuevo diario en Galicia, que hará el número trece de información general y catorce si incluimos el de información deportiva. Lo publicará Edicións de Referencia, empresa creada para editar un periódico independiente, progresista y en gallego. Con sede en Santiago, la empresa editora presentó el proyecto en febrero de 2001 en un acto celebrado en Compostela, pero todavía no ha anunciado fecha de salida a la calle.

Menos cambios ha habido en el sector de las publicaciones periódicas en papel, que ha visto como a principios del 2000 aparecía la revista mensual Imagen\&Comunicación, que en el 2001 amplió su difusión a Asturias y León. El único semanario de información general en lengua gallega, A Nosa Terra, sigue con su cita semanal con Alfonso Eiré al frente de la dirección, quien en estos últimos años ha establecido una política de diversificación empresarial que permitió el nacimiento de un grupo editorial en gallego que publica periódicamente trabajos de investigación, libros de texto y obras de divulgación.

Los últimos años del siglo XX sirvieron de marco para el nacimiento de Tempos Novos, revista mensual de información para el debate; Fadamorgana, revista de literatura infantil y juvenil trimestral que se publica desde abril de 1999; y Guía dos Libros Novos, publicación mensual sobre novedades del mundo editorial que mantiene su cita puntual todos los meses desde noviembre de 1998 . Las tres publicaciones, editadas en gallego, mantienen su cita periódica a comienzos del 2001 en un mercado donde continuan cabeceras históricas como Grial, trimestral de cultura, o A Trabe de Ouro, también trimestral de cultura.

\section{Los grupos ganan tamaño}

Las empresas editoras de diarios en Galicia no se han conformado con los cambios en la dirección, sino que han impulsado proyectos de reestructuración para el diseño de nuevos modelos. Es el caso de La Voz de Galicia, grupo líder, 
que estableció el número de ediciones en catorce (unificó las tres ediciones de Ourense -Valdeorras, Ourense y Carballiño-Ribeiro- en una provincial) y reorganizó sus cuadros directivos, situando a Manuel Areán Lalín como secretario general.

El cambio en la responsabilidad editorial fue el primer paso para que Emilio Rey Berguer dejase oficialmente el puesto de consejero delegado y el grupo, hecho que se produjo oficialmente el 28 de febrero de 2001. El presidente del Grupo Voz, Santiago Rey Fernández-Latorre recuperó a final de febrero el control de La Voz de Galicia y apartó a sus dos hijos de los cargos de vicepresidente (Santiago Rey Berguer) y consejero delegado (Emilio Rey Berguer) que ocupaban hasta ese momento.

El Grupo Voz, propietario de Diario 16 y Diario de León, decidió que la responsabilidad editorial de estas cabeceras la asumiesen también las respectivas sociedades (desde febrero de 2001). En este año cerró Diario 16. El grupo mantiene su presencia en el sector audiovisual, a través de Noroeste de Radio Televisión (NTR), de la que dependen NTR Ficción, NTR Telefábrica y NTR Noticias, y a través de Atlas Galicia, la cadena que Telecinco y el Grupo Voz crearon en 1998. En radio, desde 1999, Radio Voz se integró en el proyecto radiofónico de Telefónica Media, pasando a gestionar 36 frecuencias en Galicia con tres programaciones: Onda Cero, Radio Voz y Europa FM. El Grupo Voz también participa con un 15 por ciento del capital en Telefónica Cable de GaliciaVER

El segundo grupo que diseñó operaciones a corto y largo plazo en el panorama gallego ha sido El Progreso, que aumentó su presencia en el mercado con la adquisición de Diario de Pontevedra (en 1999), con el control de la mayoría de las acciones y la gestión desde el año 2000 de la Axencia Galega de Noticias (AGN), la agencia de noticias privada promovida por los pequeños diarios, y con la potenciación de Telelugo, una televisión local para Lugo.

Otro grupo que ganó en tamaño ha sido La Capital, que inicialmente editaba El Ideal Gallego, más tarde compró Deporte Campeón y que ahora, además de estas cabeceras, cuenta con Diario de Ferrol (desde 1999) y que en febrero de 2001 puso en la calle Diario de Arousa. La Capital también dispone de una red de publicaciones comarcales que adquirió en el año 2000 al grupo Filmagal.

También Prensa Ibérica, de la mano de La Opinión de A Coruña, puso en marcha un diario para entrar en un mercado local donde el Grupo Voz tiene su sede central y en una ciudad donde La Voz de Galicia vende la mayor parte de los ejemplares que difunde. Faro de Vigo, la principal cabecera en Galicia de Prensa Ibérica, orientó su diversificación hacia el cable, donde participa entre los promotores del Grupo Gallego de Cable (Grupo R), que lidera Unión Fenosa y Caixanova como adjudicatario de las tres demarcaciones de Galicia. Desde 1998 también están adheridos a este grupo las editoras de los diarios El Progreso, La Región, El Correo Gallego y El Ideal Gallego. 
El Grupo Correo Gallego, que tiene en Editorial Compostela la empresa que edita, imprime y distribuye los diarios El Correo Gallego y O Correo Galego, éste último el único que se edita en la lengua de Rosalía de Castro, cuenta con Edicións Correo como empresa que gestiona la edición y composición de textos de publicaciones periódicas no diarias, boletines oficiales y libros (en el año 2001 edita el Diario Oficial de Galicia), y con Telecable Compostela, que gestiona la explotación de emisoras de radio en frecuencia modulada (Radio Obradoiro), así como la producción y grabación de producciones audiovisuales ("Pratos Combinados", serie de ficción para Televisión de Galicia).

El Grupo La Región, además de un canal de televisión local, Telemiño, que emite en Ourense, dispone de otra marca para Vigo, Telecíes, y mantiene la mayoría de las acciones de Atlántico Diario, que se edita en Vigo, y la edición de La Región Internacional, que publica con periodicidad semanal. La producción de televisión la realiza a través de LR TV Madrid y LR TV Ourense.

Los principales grupos de comunicación gallegos que cuentan con medios impresos disponen de plantas de impresión. Es el caso de La Voz de Galicia, Faro de Vigo, El Correo Gallego, El Progreso, La Región, El Ideal Gallego y Diario de Pontevedra. Otras dos plantas de impresión existentes en territorio gallego pertenecen al grupo Prisa, que cuenta desde noviembre de 1997 con Norprensa en Lugo, en el Polígono de O Ceao, donde se imprime El País, Cinco Días y As, y del Grupo Recoletos, que tiene Recoprint Rábade en la localidad lucense de Rábade, donde imprime los diarios Marca y Expansión, además de suplementos para estos diarios.

\section{La expansión multimedia}

La política de los grupos de comunicación gallegos, casi todos nacidos alrededor de un diario familiar, busca la diversificación para establecerse en el nuevo modelo empresarial: los multimedia. Los medios gallegos permanecen dentro de la tendencia general, con estrategias de atención a la información de proximidad para dificultar la entrada de los medios de fuera en lo que consideran sus mercados y de alianzas con grupos de otros ámbitos (en prensa, sólo Faro de Vigo y La Opinión A Coruña pertenecen a una cadena estatal, Prensa Ibérica).

Los pasos dados para la estrategia multimedia han permitido advertir los puntos débiles de las empresas gallegas, que tienen que ver con la financiación, ya que para el crecimiento apenas encuentran otros recursos distintos de los créditos. La deuda a corto plazo ha pasado de 11.380 millones en 1996 a 13.719 millones en 1997 -en 1989 el endeudamiento total de la prensa gallega era de 5.815 millones-, mientras la difusión fue semejante en 1998 a la registrada en 1997, con 225.519 ejemplares frente a 225.999.

La difusión en 1999 y en 2000 muestra la tendencia a la estabilidad en un mercado donde es muy difícil conseguir nuevos lectores y donde las cabeceras se 
pelean por conquistar lectores de la competencia. La Voz de Galicia cuenta con la mitad del mercado de la prensa diaria editada en Galicia.

Evolución de la difusión de la prensa gallega 1999-2001

\begin{tabular}{|lcccc|}
\hline XORNAIS & $\mathbf{1 9 9 9}$ & $\mathbf{2 0 0 0}$ & $\mathbf{2 0 0 1}$ & Evolución \\
\hline La Voz de Galicia & 108.841 & 107.850 & 108.201 & -640 \\
\hline El Correo Gallego & 18.126 & 18.238 & 22.735 & 4.609 \\
\hline El Progreso & 15.104 & 15.526 & 15.610 & 506 \\
\hline La Región & 12.433 & 12.844 & 12.433 & 0 \\
\hline Faro de Vigo & 42.278 & 42.639 & 42.913 & 635 \\
\hline Atlántico Diario & 4.146 & 4.345 & 4.146 & 0 \\
\hline Diario de Pontevedra & Sin control de OJD & 5.582 & 6.332 & 750 \\
\hline
\end{tabular}

Fuente: OJD

La evolución del pasivo en la empresa periodística gallega durante la década de los noventa tiene dos picos altos en 1992 y en 1997. Las estimaciones del valor de las principales empresas periodísticas que editan diarios en Galicia alcanzan una estimación de más de cien mil millones de pesetas. Por su parte, el capital social del conjunto de las empresas periodísticas gallegas ronda los dos mil quinientos millones de pesetas ${ }^{1}$

Según los datos correspondientes a 1999 depositados en el registro mercantil, los beneficios de las siete principales empresas editoras de Galicia sumaban 2.343.328 millones de pesetas, los ingresos superaban los veintiún mil millones y el personal ocupado los mil cien trabajadores. El total de inmovilizado neto se aproxima a los doce mil millones y el activo circulante los trece mil millones. El total del activo y el pasivo está en los veinticinco mil millones de pesetas, mientras los ingresos se sitúan entre las 520.304 .000 pesetas de Rías Baixas y las 10.994.238.000 pesetas de La Voz de Galicia (Editorial Celta, 736.569.000; El Progreso, 1.646.448.000; La Región, 1.975.029.000; Editorial Compostela, 5.322.083.000; y Faro de Vigo, 3.551.418.000 pesetas), según los datos del ejercicio de 1999 depositados en el Registro Mercantil.

1 Las estimaciones se basan en los estudios del profesor Francisco Campos en su tesis doctoral sobre $A$ reconversión da prensa en Galicia 1982-1992 y los trabajos posteriores del mismo autor sobre la evolución económica de las empresas periodísticas gallegas. 
Beneficio neto de las empresas editoras de la prensa gallega. 1999.

\begin{tabular}{|lc|}
\hline Periódicos & Año 1999 \\
\hline $\begin{array}{l}\text { RIAS BAIXAS, S. A. } \\
\text { ATLÁNTICO DIARIO }\end{array}$ & 17.163 .000 Ptas. \\
\hline $\begin{array}{l}\text { EDITORIAL COMPOSTELA, S.A. } \\
\text { EL CORREO GALLEGO }\end{array}$ & 34.116 .000 Ptas. \\
\hline $\begin{array}{l}\text { EL PROGRESO, S.L. } \\
\text { EL PROGRESO }\end{array}$ & 73.804 .000 Ptas. \\
\hline $\begin{array}{l}\text { FARO DE VIGO, S.A. } \\
\text { FARO DE VIGO }\end{array}$ & 460.084 .000 Ptas. \\
\hline $\begin{array}{l}\text { LA REGION, S.A. } \\
\text { LA REGION }\end{array}$ & 1.445 .830 Ptas. \\
\hline $\begin{array}{l}\text { LA VOZ DE GALICIA, S.A. } \\
\text { LA VOZ GALICIA }\end{array}$
\end{tabular}

Fuente: Datos depositados por las empresas en el Registro Mercantil

Las cifras muestran los puntos fuertes y débiles de sus economías. Es decir, reflejan las limitaciones para intervenir con planes ambiciosos en el actual escenario de medios, donde hay una clara tendencia a la concentración, a las alianzas y a la creación de grupos estables, capaces de competir sin aumentar excesivamente el endeudamiento. Y las subvenciones flotan en un ambiente mediático en busca de nuevos horizontes no siempre bien definidos.

En cambio, lo que mantiene bastante bien la prensa gallega es la mayoritaria difusión de sus productos en el territorio de la Comunidad autónoma frente a los medios de fuera (especialmente de Madrid). Para ello, la prensa ha puesto en marcha estrategias de atención a lo próximo, con la creación de ediciones y páginas con información de las principales localidades, y de importantes cambios en los contenidos. Así podemos decir que la presencia de la información política, que en 1978 representaba un 49 por ciento de todos los textos publicados, en 1998 era del 24 por ciento. Y en 2001 se estima que no alcanza el 20 por ciento. 


\section{Evolución temática de la prensa gallega 1978-1998}

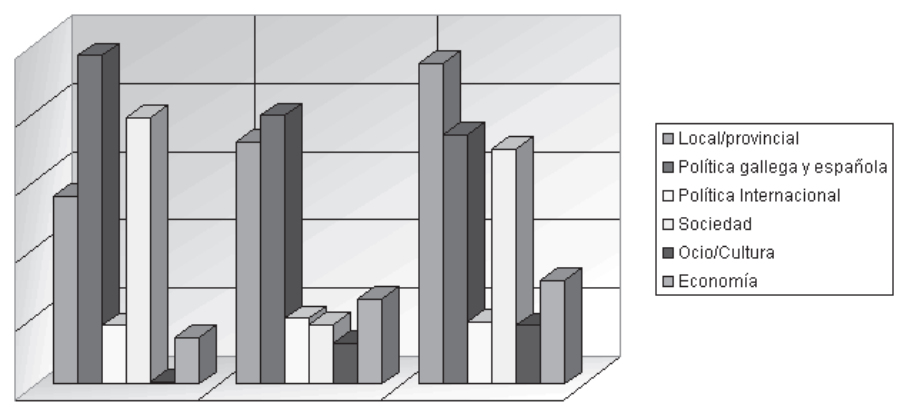

Fuente: Grupo de Investigación de Medios Impresos y Electrónicos de la Facultad de Ciencias de la Información de la Universidad de Santiago de Compostela.

\section{Las versiones digitales}

Lo que han potenciado en el último año las editoras de diarios ha sido su versión digital. Faro de Vigo implantó en julio de 2000 un sistema de edición para publicar en la red más noticias cada día. El Progreso Digital, que se estrenó en la red a mediados de 2000, renovó el diseño y aumentó la información en enero de 2001. Diario de Pontevedra cambió su página en 2000. La Región Internacional abrió en Internet un portal con información útil para el emigrante en abril de 2000.

En la primera quincena de mayo de 2000 El Ideal Gallego abrió página web, mientras el día 17, <Día das Letras Galegas>, fue la fecha elegida por La Voz de Galicia para lanzar su versión digital del diario en Internet. Canal Voz, la sociedad de contenidos digitales nacida en 1999, apareció en el mercado para ofrecer respuestas estratégicas a las demandas de mercado de la nueva economía. Y el 1 de agosto de 2000 apareció la versión digital de Diario de Ferrol, que con su iniciativa hizo que todos los diarios de información general de Galicia tuviesen edición digital.

En enero del año 2000 apareció el primer diario de Galicia que sólo se publicaba en línea: Xornal.com, promovido por el periodista José Luis Gómez, ex director de La Voz de Galicia y actual integrante del equipo de dirección del Grupo Zeta como director de Coordinación Editorial y de Comunicación. El año 2000 también llevó a Internet al segundo diario que sólo se publica en línea, EGalicia Diario Digital, en la dirección electrónica Celtanet.com, que apareció el día 18 de febrero.

Otra página web veterana y varias veces premiada es la de la Compañía de Radio Televisión de Galicia (CRTVG), que ya superó los cien millones de visitas 
en octubre de 2000 y el 31 de enero de 2001 ya había rebasado la barrera de las ciento cincuenta y seis millones de visitas. En mayo de 2002 el número de accesos superaba los 462 millones. Se trata de una web que, bajo la dirección de Alberto Barciela, tiene páginas en nueve idiomas (gallego, castellano, italiano, inglés, euskera, catalán, portugués y francés). El Correo Gallego, primer diario de Galicia que contó con versión digital, renovó diseño y contenidos en 2000 para reforzar su apuesta por Internet.

De las últimas apariciones de productos gallegos en la red hay que destacar el portal U-lo, que comenzó en septiembre de 2000, y el portal del Consello da Cultura Galega (Culturagalega.org), que despidió el año con su estreno en la red (el 10 de octubre de 2000) y con el reconocimiento del premio Nuevos Lenguajes para Galicia y el premio al mejor producto en Internet 2000 Nuevos Lenguajes (fue la segunda iniciativa más innovadora del año en España según los premios que promueve la Fundación Coca-Cola). En junio de 2002 estrenó nueva imagen.

A pesar de estas iniciativas innovadoras, Galicia está por debajo de las medias nacionales en equipamiento informático y ordenadores (también en consumo de prensa, lectura de libros, bibliotecas públicas,...). Los datos dicen que el país gallego aparece situado en los lugares más bajos de acceso y uso de nuevas tecnologías, por lo que existe el convencimiento de que es necesario un esfuerzo general y planificado hacia el desarrollo de nuestras posibilidades en la nueva realidad que se anuncia ${ }^{2}$.

\section{Una radio en cadena}

La radio festejó en 2000 los quince años de la Radio Galega, la cadena autonómica que dirige desde octubre de 1999 Alfonso Cabaleiro. Este año, el de 1999, la Cope nombró nuevo director para Galicia a Fernando Macías. En junio de 2000 fue Cope Lugo la emisora que renovó equipo con la designación de Arturo Cerviño como director. Los cambios en la radio de Galicia prosiguieron en 2001, en enero, con el nombramiento de Xosé Luis Blanco Campaña, primer director de la Radio Galega, como nuevo director de RNE en Santiago de Compostela, y en marzo, mes en el que Ramón Blanco se hizo cargo de la dirección de Radio España en Galicia en sustitución de Javier García Sánchez.

El sistema radiofónico gallego en tecnología analógica lo integran doscientas treinta y tres emisoras públicas y comerciales, de las que ciento veinte corresponden al sector público y ciento once al privado, además de una de carácter

2 Víctor Fernández Freixanes, profesor de la Facultad de Ciencias de la Información de Santiago, y el empresario Lois Rodríguez, director de Item:)Aga, estiman, en su capítulo sobre Nuevos Medios del informe A Comunicación en Galicia 2000, que Galicia debe entrar en la Sociedad de la Información equipada en condiciones de competitividad y suficiencia para acceder a las nuevas vías de información y conocimiento que se anuncian. 
educativo. Es un sistema que se multiplicó por ciento en los últimos veinte años, ya que en 1982 sólo había en Galicia cuarenta y seis emisoras y fueron las concesiones de nuevas frecuencias por parte de la Xunta, en 1983, 1989 y 1998, las que hicieron posible la ampliación de ciento ochenta y seis nuevas frecuencias ${ }^{3}$.

A partir de dos de las últimas concesiones de frecuencias moduladas ha nacido el grupo privado radiofónico gallego que busca la consolidación de una cadena privada de programación propia las veinticuatro horas: Cadena Noroeste (Cadena Noroeste de Medios de Comunicación, S.A.). Los acuerdos con distintas radios locales permitieron a este grupo la creación de una red de emisoras que el 4 de mayo de 2001 inició las emisiones.

Las emisoras públicas y privadas de ámbito gallego y local conviven con treinta y una emisoras municipales, agrupadas en la asociación Emisoras Municipais Galegas (EMUGA), que mantiene una importante actividad desde finales de los ochenta, sobrepasó la treintena a finales de la década de los noventa y continúa como un grupo radiofónico relativamente estable de comunicación local.

La mayor parte de las emisoras públicas y privadas emiten programación en cadena y cuentan con desconexiones locales o autonómicas. Y los datos apuntan que cuentan con una audiencia que se ha estabilizado desde 1991. Las emisoras gallegas contaban en el año 2000 con 1.215 .598 oyentes diarios, resultado de la actitud receptiva de 938.970 gallegos -el $34,2 \%$ de la población- que escuchan cada día una emisora de radio. ${ }^{4}$

\section{Cambios en televisión}

Uno de los sectores donde se apuntan más cambios en el horizonte es en el audiovisual, que en los últimos años se ha mostrado especialmente dinámico en Galicia y que ahora espera la digitalización, con la correspondiente concesión de licencias, para iniciar una nueva transformación. En 1999, las productoras gallegas participaron en ocho películas de cine que tuvieron Galicia como principal centro de realización. En el 2000 la cifra permaneció invariable.

Televisión de Galicia y el Centro Territorial de RTVE en Galicia, situados en el monte compostelano de San Marcos, constituyen el epicentro del sistema audiovisual gallego, que representa un volumen de negocio total de unos treinta mil millones de pesetas y casi tres mil empleos directos ${ }^{5}$. La principal oferta

3 Los datos proceden del último informe sobre A Radio del profesor Xosé Ramón Pousa, elaborado para el informe A Comunicación en Galicia 2000, editado por el Consello da Cultura Galega.

4 El número de oyentes de radio en Galicia figura en los estudios de audiencia realizados por el equipo de investigación que dirige el profesor Xosé Ramón Pousa.

5 La evolución del sector audiovisual gallego lo ha estudiado el profesor Fancisco Campos en el capítulo "Galicia.com" publicado en el libro Galicia 2020, editado por Ir Indo en el año 2000. 
televisiva lleva el sello de TVG, que cuenta con una programación íntegramente en gallego durante las veinticuatro horas.

Televisión de Galicia, que incorporó a Julio Rodríguez como subdirector de informativos a final de 1999 -el único cambio significativo en los puestos directivos en los tres últimos ejercicios- y cumplió quince años en 2000, actúa como motor del audiovisual a pesar del plan de saneamiento económico con que cuenta. La Compañía de Radio-Televisión de Galicia (CRTVG) tenía una deuda de 9.200 millones de pesetas en 1994 y en la actualidad ha conseguido rebajarla de los cuatro mil millones. Las cuentas auditadas de la CRTVG detectaron en 1994 una deuda acumulada de 9.2000 millones, que se refinancia con tres créditos bancarios (BSCH, Caixa Pontevedra y Caixa Vigo) que suman 7.268 millones de pesetas. Los 1.600 millones restantes se reconvierten en deuda a corto plazo y se van refinanciando y amortizando año tras año. El plan de saneamiento apuntaba que el 30 de julio de 2001 la deuda estaba en 3.858 millones de pesetas y preveía que en siete años quede totalmente amortizada ${ }^{6}$.

Televisión de Galicia destinó en el año 2000 directamente a coproducción de películas (ocho largometrajes), documentales, series y producciones del audiovisual gallego alrededor de cuatro mil quinientos millones de pesetas. La apuesta por el sector audiovisual ha coincidido con un incremento de audiencia del canal público autonómico, que en el año 2000 ha tenido una cuota media de pantalla del $19,0 \%$ en el total día y del $22,4 \%$ en la franja de las $20: 30$ a 24 horas, lo que ha supuesto la superación de todos los registros alcanzados en la historia de Televisión de Galicia. Esta tendencia tuvo continuidad entre el 1 de enero y el 27 de febrero de 2001, período en el que mantuvo constante el incremento de audiencia.

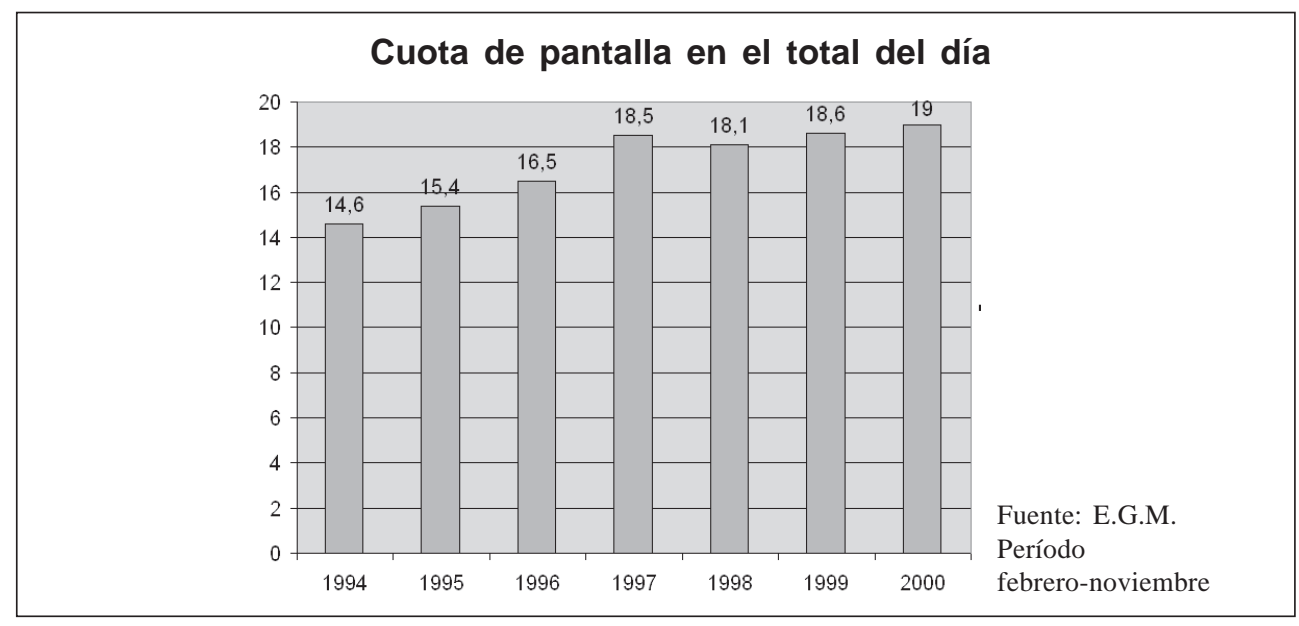

6 Los informes de gestión y cuantas anuales de Televisión de Galicia de los últimos tres años aportan estos datos sobre la situación económica y la amortización de la deuda. 


\section{Estudio General de Medios. Cuota de pantalla}

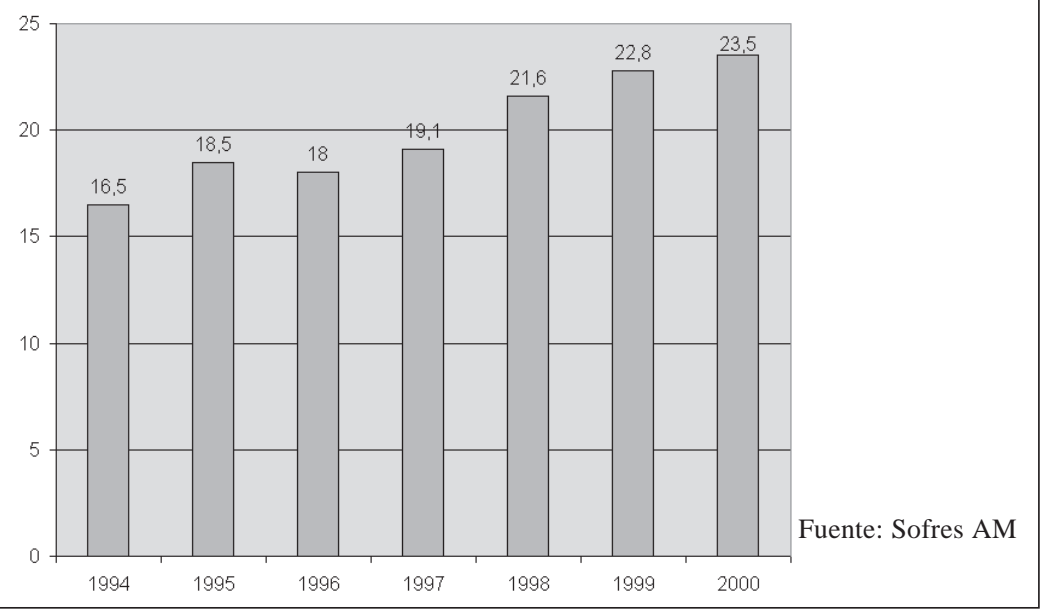

En el ámbito de Galicia, los datos de audiencia de 2000 situaron a Televisión Española como líder, con un $27,3 \%$ de la cuota en el total del día. Tele 5 ocupó la segunda posición, con una media del 21,2\% y Antena 3 la tercera posición, con un 20\%, por encima de Televisión de Galicia, que alcanzó una cuota media de pantalla del $19 \%$.

La otra televisión pública, Televisión Española, líder de audiencia, como hemos dicho, amplió en marzo de 1999 su programación para Galicia de la mano de convenios firmados con la Xunta de Galicia. Pasó de una hora al día de producción propia (media hora en cada cadena) a dos horas y media diarias (media hora en la primera cadena y dos horas en la segunda cadena). El centro regional, que tiene a Octavio Rodríguez como director desde julio de 1998, ha dado, pues, pasos para aumentar su oferta y competir con Televisión de Galicia y con las televisiones privadas.

El sector, además de TVG, TVE en Galicia, Atlas Galicia-Telecinco y Antena 3 Galicia, está formado por más de noventa medianas y pequeñas empresas, más de cuarenta integradas en la Asociación Galega de Productoras Independentes (AGAPI), que en 2002 tiene a Valentín González como presidente. No obstante, el censo de empresas en funcionamiento efectivo asciende a 74 sociedades, de las que cerca de cincuenta cuentan con actividad estable?

Telecinco, la primera televisión privada que inició desconexiones de informativos diarios en Galicia, en mayo de 1998, amplió las emisiones específicas

7 Los datos de empresas en funcionamiento efectivo proceden de la base de datos del Observatorio do Audiovisual, creado y coordinado por Margarita Ledo, catedrática de Comunicación Audiovisual de la Universidad de Santiago de Compostela. 
para Galicia en mayo de 1999, hasta que cambió de opinión y cortó las desconexiones para los informativos diarios de Galicia el 1 de agosto de 2001. Antena 3, que disponía de un programa informativo semanal, Galicia a fondo, desde marzo de 1996, apostó por las desconexiones de informativos diarios desde el 25 de julio de 1999 y mantuvo Galicia a fondo. En 2002 mantenía un informativo de lunes a viernes de media hora de información de Galicia.

En este panorama audiovisual de incremento de la producción propia para Galicia también aparecen nuevos actores. Los datos indican que surgen nuevas iniciativas, sobre todo pequeñas productoras, y proyectos ambiciosos en el campo de la animación. Es el caso de Brent Entertaiment. A principios de 2000 se inauguró la primera factoría de producción de animación Brent Entertaiment, con una inversión inicial de mil millones de pesetas y cerca de cien puestos de empleo directo.

El buen momento del audiovisual gallego prosiguió en los primeros meses de 2001 con el premio Goya a la banda sonora original del filme Sé quién eres, interpretada por la Sinfónica de Galicia, y con la designación de Pancho Casal, director general de la productora Continental, como ponente en el comité que participará en los trámites parlamentarios que precederán a la aprobación del Proyecto de Ley de Fomento y Promoción de la Cinematografía y el Audiovisual, más conocida como Ley del Cine. En 2002 los Goya llegaron a Galicia para O Bosque Animado.

De la misma forma, los medios audiovisuales locales viven momentos de cambios significativos. Las televisiones locales caminan hacia la constitución de grupos para mejorar su implantación territorial y su audiencia. Los primeros pasos los ha dado a comienzos de 2001 el grupo Prisa con la creación de la Unión de Televisiones Gallegas, empresa en la que participan el Banco Pastor, Asociados de la SER, Grupo Prisa y Compostela Visión, entre otros socios, y que tiene al periodista Xavier Otero como director general.

La reestructuración del grupo El Progreso afectó también a la televisión local, Telelugo, que en diciembre de 2000 estrenó directora. Margarita Soilán Carbia ocupó el puesto y el hasta ese momento director, José Luis Tejero, pasó a la dirección de producciones de Lugo Press, empresa de nueva creación para proyectos audiovisuales. De la misma forma, Telecoruña canal 41 y Televigo han tenido reestructuración en los últimos años, con cambios en la propiedad y en los puestos de máxima responsabilidad.

En total, unas veinticinco televisiones públicas y privadas forman la red básica de televisión local de Galicia que mantiene una actividad relativamente estable. Además de las citadas, destaca en la oferta de televisión local la programación de TV Ferrol Canal 31, Telesalnés y TV7 Monforte, entre las privadas, y de Ponteareas, la más veterana de las públicas locales gallegas, desde 1987, con cinco horas de programación propia diaria, y Cerceda en las públicas, con hora y media diaria de producción propia (excepto los domingos). 


\section{La publicidad}

En los últimos años en Galicia surgieron decenas de agencias de publicidad y consultorías de comunicación con el objetivo de abrir una rentable fuente de negocio en este sector, que se encuentra en expansión. Aunque el volumen de facturación de las empresas de publicidad es pequeño por ahora, lo cierto es que las expectativas son muy positivas, derivadas fundamentalmente de la alta competitividad a que se enfrenta el empresariado gallego, que está solicitando planes de comunicación, programas de identidad corporativa y mensajes publicitarios diversos ${ }^{8}$.

Según los datos de la base de datos Ardán, elaborada por el Consorcio de la Zona Franca de Vigo y el Instituto Galego de Promoción Económica (IGAPE), la primera agencia gallega de servicios de publicidad por volumen de negocio es Imaxe Publicidade, que facturó en 1999 la cifra de 1.063.810.000 pesetas, mientras que la agencia de publicidad general que ocupa el primera lugar corresponde a Grupo Bap Conde, con 545.840.000 pesetas de facturación.

\section{SERVICIOS DE PUBLICIDAD}

\begin{tabular}{|lc|}
\hline IMAXE PUBLICIDADE S.A. & 1.063 .810 .000 pesetas \\
\hline STRATEGIAS DE MEDIOS GALICIA S.A. & 796.898 .000 pesetas \\
\hline STD-Multiopción S.A. & 658.230 .000 pesetas \\
\hline MAXAN S.A. & 621.819 .000 pesetas \\
\hline $\begin{array}{l}\text { RECLAM. Publicidade, } \\
\text { marketing y artes gráficas S.A. }\end{array}$ & 583.500 .000 pesetas \\
\hline
\end{tabular}

Fuente: Ardan. Galicia Directorio e Informe Económico Financeiro.

IGAPE y Consorcio de la Zona Franca de Vigo. 2000.

AGENCIAS DE PUBLICIDAD GENERAL

\begin{tabular}{|ll|}
\hline GRUPO BAP CONDE & 545.840 .000 pesetas \\
\hline J\&J DEL RIO & 243.765 .000 pesetas \\
\hline PALACIOS PUBLICIDADE S.L. & 206.815 .000 pesetas \\
\hline COMUNICACIÓN INTEGRAL GALLEGA S.L. & 127.265 .000 pesetas \\
\hline INTERMEDIARIOS DE COMUNICACIÓN & 104.568 .000 pesetas \\
\hline
\end{tabular}

Fuente: Ardan 2000. Galicia. Directorio e Informe Económico Financieiro.

IGAPE y Consorcio da Zona Franca de Vigo. 2000.

8 El interés de los empresarios gallegos por la publicidad y las perspectivas del sector las ha estudiado el profesor Alberto Pena en el capítulo sobre A Publicidade del informe titulado A Comunicación en Galicia 2000, editado por el Consello da Cultura. 
Los datos indican que la mayoría de las cien agencias registradas actualmente en Galicia dedicadas a la comunicación comercial nacieron en la última década -la más antigua es Reclam Publicidad, Marketing y Artes Gráficas, fundada en 1939 en A Coruña por Domingo Torrón. Las ciudades elegidas para sede central de estas nuevas agencias son Vigo, A Coruña y Santiago de Compostela, seguidas de Lugo, Pontevedra, Ourense y Ferrol. Y casi todas estas empresas de publicidad ofrecen servicios plenos a sus clientes.

De los estudios realizados por Alberto Pena en los últimos años se deduce que los anunciantes gallegos que más utilizan los servicios de agencias publicitarias pertenecen al sector de la automoción (concesionarios de coches, fábricas auxiliares de automoción o marcas consolidadas como Citroën) y a la alimentación (grandes superficies comerciales como Alcampo o Continente y productos pesqueros, entre otros). A continuación figuran los fabricantes de telefonía, de productos textiles y de tecnología aplicada.

\section{Infraestructura tecnológica}

Los esfuerzos para dotar a Galicia de infraestructura tecnológica y conseguir que salga de los últimos puestos de acceso y uso de nuevas tecnologías se han multiplicado en los últimos años. Más de trescientos mil gallegos estaban conectados a Internet a comienzos de 2001 y las previsiones apuntan que pronto se multiplicarán a medida que $\mathrm{R}$ aumente el cableado en las ciudades de Galicia y Telefónica aplique su proyecto de mejora de infraestructura y de llevar la alta velocidad por red a todo el territorio de la comunidad.

Para potenciar la infraestructura de telecomunicaciones, Galicia dio un importante paso con la creación de la Autopista Galega da Información (AGI), que tiene fecha de 1997 (el 3 de octubre se inauguró), cuando un acuerdo entre la Xunta y la empresa Fujitsu permitió la instalación en el corazón de la red de un conmutador de ATM de altas prestaciones Fetex 150, capaz de llegar hasta los $150 \mathrm{Gbps}$, que tiene las funciones de nodo central de la red. A comienzos de 2001 cuenta con más de veinticinco puntos de acceso (los siete campus de las tres universidades gallegas, los principales hospitales, el Parque Tecnológico de Ourense, el Centro Superior Bibliográfico y el Centro Ramón Piñeiro, entre otros).

En el momento actual la red da servicio de transmisión de datos, voz y vídeo a los centros conectados. Además, con la finalidad de avanzar en la implantación de las telecomunicaciones en todos los ámbitos de la sociedad gallega, se realizan aplicaciones de teleenseñanza, acceso a bibliotecas, telemedicina, red IRIS en Galicia y servicios portadores de circuitos de datos. Conectada a la AGI también funciona una red de seis centros multimedia para promover la teleenseñanza y la formación de los ciudadanos en las tecnologías actuales, que aspira a cubrir todo el territorio con salas multimedia en todas las comarcas. 


\section{La profesión se colegia}

Los periodistas gallegos despidieron el siglo XX con iniciativas para poner fin a la escasa actividad de sus numerosas asociaciones profesionales (asociaciones de la prensa y otras entidades asociativas) y para iniciar la vía de la colegiación profesional. El Parlamento autonómico aprobó por unanimidad de todos los grupos (Popular, Nacionalista, Socialista y Mixto) la Ley de Creación del Colegio Profesional de Periodistas de Galicia, publicada en el Diario Oficial de Galicia el 17 de julio de 2000.

Fue el primer paso para la convocatoria de elecciones del primer Decano del Colegio, puesto al que aspiraron Xosé María Palmeiro y Arturo Maneiro. Venció el primero por 482 votos frente a los 180 que recibió Arturo Maneiro y una abstención del 35 por ciento de los más de mil periodistas asociados. Xosé María Palmeiro y su equipo iniciaron la aplicación del programa de gobierno de la nueva corporación en los últimos meses de 2000.

En el nuevo escenario de la Sociedad de la Información, la mayoría de los periodistas gallegos optaron por el Colegio Profesional, ya que el primer censo confeccionado incluye un total de 1955 personas que habitualmente se dedican a la elaboración de información en Galicia. Aunque no se trata de un censo cerrado, lo cierto es que refleja a los hombres y mujeres que de una forma habitual y constante trabajan como periodistas ${ }^{9}$.

El perfil del periodista gallego es un hombre (el 57,7 por ciento), menor de 30 años (el 46 por ciento), licenciado en periodismo (65,7 por ciento), trabaja en Santiago de Compostela (el 38,6 por ciento), en el medio prensa (43,3 por ciento) y habla castellano (el 50,3 por ciento). En cuanto a las condiciones laborales, el 58 por ciento tiene contrato fijo, contrato como redactor (el 70,4 por ciento), jornada media de 8,4 horas al día, cobra 1.170 pesetas brutas a la hora (1.330 los hombres y 995 las mujeres) y está satisfecho del trabajo realizado (el 86,67 por (iento) ${ }^{10}$.

\section{El marco normativo}

El marco normativo que regula el mundo de la comunicación registró dos importantes incorporaciones: la Ley de Creación del Colegio Profesional de Periodistas, ya citada en el apartado anterior, y la Ley del Audiovisual de Galicia. Las dos consiguieron el apoyo unánime de la Cámara autonómica, lo que supuso un claro apoyo de todas las formaciones políticas a la organización de los periodistas

9 El primer censo de periodistas de Galicia lo hizo en el año 1999 un equipo dirigido por el profesor Miguel Túñez como parte del trabajo previo a la elaboración del estudio sobre las condiciones laborales y actitudes profesionales en los medios de comunicación.

10 Las características del perfil del periodista y las condiciones laborales destacan entre las conclusiones del estudio sobre Condicións laborais e actitudes profesionais nos medios de comunicación, editado por el Consello da Cultura en el año 2000 y coordinado pro Miguel Túñez y X. López. 
y a la consideración del audiovisual como un sector industrial estratégico de Galicia.

La Ley del Audiovisual, de 1 de septiembre de 1999, obligó a un nuevo decreto de regulación de la composición y funciones del Consello Asesor de Telecomunicacións e Audiovisual de Galicia, de octubre de 1999. El nuevo marco normativo potencia el papel del Consejo Asesor con la posibilidad de crear comisiones sectoriales de operadores, usuarios y empresas relacionadas con las actividades audiovisuales. Entre sus funciones figura la elaboración de informes sobre los temas relacionados con las telecomunicaciones y el audiovisual, que el presidente del Consejo someta a su consulta, así como la resolución mediante el procedimiento del arbitraje de los conflictos que le sometan a consideración los interesados.

Para establecer el reglamento del funcionamiento del Consejo Asesor, el conselleiro de Cultura, Comunicación Social y Turismo dictó una orden del 17 de noviembre de 1999 que buscaba una mayor eficacia de este organismo. No obstante, a pesar del nuevo marco normativo, todavía no ha conseguido el impulso que los actores del sector audiovisual esperaban después de la aprobación por unanimidad de la nueva ley.

\section{Los desafíos}

A partir de los datos de 1999, 2000, $2001 \mathrm{y}$, en algunos casos, 2002, citados en este artículo, podemos asegurar que el hipersector de la comunicación en Galicia tiene importantes retos que vencer. Luces y sombras se mezclan en un escenario donde hay muchos sueños, algunas expectativas y un número muy concreto de realidades.

El punto de partida para el nuevo milenio parece claro: Galicia ha entrado en el siglo XXI con un sistema de medios de comunicación que se caracteriza por el predominio de la empresa de propiedad familiar, por una cierta debilidad empresarial (incremento de la deuda y dependencia de las subvenciones), por el liderazgo en el mercado local y regional frente a los medios de Madrid, por una estrategia de atención a los contenidos de proximidad y por la escasa presencia de la lengua propia en los contenidos de la mayoría de los productos de las empresas privadas.

El modelo gallego de medios de comunicación se caracteriza por el acentuado carácter local, especialmente en los medios impresos, y con un importante predominio de la información propia frente a la información producida en el exterior o por empresas del exterior. Las televisiones privadas y las cadenas de radio constituyen los medios con menos información propia.

El escenario dibuja un mapa muy definidio de medios: es un modelo paradigmático de medios locales que a lo largo de la historia ha conseguido sentar las bases para articular una fuerte implantación en los ámbitos de proximidad y 
una aceptable sintonía con los usuarios en un mercado presidido por una tendencia a la estabilidad (los índices de difusión y audiencia se han estabilizado desde 1995).

En los últimos años hubo importantes cambios en la mayoría de las empresas, que han aprovechado la entrada en el milenio para definir nuevas estrategias para el mercado. En el horizonte inmediato hay muchos desafíos, bastantes sueños y algunas realidades. El sector está en el inicio de una importante reestructuración que puede conducir a la consolidación de un modelo propio dominado por empresas familiares de Galicia o a una mayor entrada de los grandes grupos de comunicación con presencia en el Estado. De momento la empresa de comunicación gallega sigue siendo familiar, la dimensión de las empresas pequeña y mediana, cierta debilidad empresarial, la diversificación está en fase inicial, el modelo de medio local o multilocal y el idioma mayoritario en los productos informativos es el castellano. Estas son algunas de las realidades, que se mezclan con los sueños de un futuro mejor.

\section{Referencias Bibliográficas:}

- BORJA, Jordi/CASTELLS, Manuel: Local y global. La gestión de las ciudades en la era de la información. Madrid, Taurus, 1994.

- CAMPOS, Francisco: A reconversión da prensa en Galicia (1982-1992). Santiago de Compostela, Universidad de Santiago, 1994.

- CAMPOS, Francisco: "Galicia.com” en ALAMO, J. CARLOS DEL y OTROS: Galicia 2020, Ir Indo, Vigo, 2000.

- CASTELLS, Manuel: La ciudad informacional. Tecnologías de la información, reestructuración económica y el proceso urbano-regional. Madrid, Alianza Editorial, 1995.

- DE PABLOS, José Manuel: Amarillo en prensa. Santa Cruz de Tenerife, Ediciones Idea, 1997.

- DÍAZ NOSTY, Bernardo: Informe anual de la Comunicación 2000-2001. Grupo Zeta, Barcelona, 2001.

- GUILLAMET, Jaume: La premsa comarcal. Un model català de periodisme popular. Barcelona, Departament de Cultura de la Generalitat de Catalunya, 1983.

- LEDO ANDIÓN, Margarita: O diario postelevisivo. Santiago de Compostela, Edicións Lea, 1993.

- LÓPEZ, Xosé: Detrás da prensa popular. Santiago de Compostela, Edicións Lea, 1995.

- LÓPEZ, Xosé (Coordinador): A comunicación en Galicia 2000. Consello da Cultura Galega, Santiago de Compostela, 2000. 
- MACIÁ MERCADÉ, Juan (1993): La comunicación regional y local. Madrid, Editorial Ciencia 3, 1993.

- MATEO Rosario DE/CAMPOS, Francisco: Comunicación rexional en Europa. Santiago de Compostela, Edicións Lea, 1997.

- MATTELART, Armand: La mundialización de la comunicación. Barcelona, Paidós, 1988.

- MORAGAS, Miquel DE/PRADO, Emilio: La comunicación local en Cataluña (1975-1988). Barcelona, Diputació de Barcelona, 1988.

- REIG, Ramón: Medios de Comunicación y poder en España. Barcelona, Paidós, 1999.

- TUÑEZ, Miguel/LÓPEZ, Xosé: Condicións laborais e actitudes profesionais nos medios de comunicación. Consello da Cultura Galega, Santiago de Compostela, 2000.

(Recibido el 20-6-2002, aceptado el 5-7-2002) 